\title{
A Method of Measuring the Sensitivity of Trypanosomes to Acriflavine and Trivalent Tryparsamide
}

\author{
BY P. J. WALKER \\ Division of Chemotherapy and Parasitology, National Institute for \\ Medical Research, London, N.W. 7*
}

(Received 6 September 1965)

\begin{abstract}
SUMMARY
Acriflavine-induced photosensitivity has been used to measure resistance in trypanosomes to certain aromatic arsenical drugs. Trypanosomes were incubated in a logarithmic dilution series of acriflavine and slides were prepared. After a standard exposure to light the \% mortality as judged by mobility was calculated and converted into probits. Mean sensitivity and its standard deviation were estimated from graphs of probits $v s \log$ concentration of acriflavine. It is possible by a graphical method of analysis to distinguish between two or three populations even when these occur in the same infection. Numerical estimates of replicate analyses and analyses made on the same strain over a period of $2 \frac{1}{2}$ years show that the system of measurement is stable. A range of $0.12 \mathrm{log}$ units has been found in all measurements made over the period on the normal strain of Trypanosoma brucei. This may be compared with the lowest degree of stable resistance which is an increase of $1 \mathrm{log}$ unit from the normal strain. Some factors which affect photosensitivity were examined, including temperature, action spectrum, concentration of drug, concentration of trypanosomes, time of incubation and the effect of oxygen. The method described is thought to be a compromise between that which is theoretically desirable and that which is simple to perform while still having an accuracy necessary for detailed studies of the development of drug resistance and of inheritance in trypanosomes.
\end{abstract}

\section{INTRODUCTION}

The discovery of the pathogenicity of African trypanosomes in the first years of this century led to a decade in which important aspects of the biology of the parasites were worked out. Many other features eluded workers then and, despite some intensive investigations in the intervening years, are still unknown. We have no certain knowledge, for example, of sexuality, of the details of the division cycle, of the mechanisms for inheritance or even of the site(s) of reproduction in the mammal. Two reasons for our deficiencies are clear. First, trypanosomes unlike bacteria do not grow readily in artificial culture, and when they do grow they change from the blood form to the insect form. Secondly, no reliable quantitative genetic characters have been found nor have accurate methods been available to assess characters such as drug resistance. Without satisfactory culture techniques it is difficult to obtain data on the division cycle, on nutritional requirements to develop gene maps and on the response of individuals to the action of drugs. The

* Present address: Zoology Department, Exeter University, Devon. 
best that has been done is to study the action of drugs on trypanosomeinfections in vivo and in vitro, as a part of the search for new chemotherapeutic agents. In these studies resistance has been found to develop readily to most drugs. In the absence of reliable visual characters, drug-resistance characters offer an alternative approach. However, the established methods for drug testing give little information about the attributes of individual trypanosomes which comprise an infection. For example, when mice are infected with 10 million sensitive parasites and 10 resistant ones the course of infection differs little whether or not the mice receive drug treatment (Hawking \& Walker, 1966). These matters have been discussed at length by Walker (1964).

A drug test method, if it is to be of use in genetical analysis, can be judged on five criteria. These are: (1) it should be sensitive and reproducible; (2) it should yield a mean value and distribution of sensitivity; (3) it should give comparable results over a wide range of sensitivity; (4) it should give data from which to distinguish and make estimates on two or more populations present in the same infection; (5) it should be applicable to drugs of different types. The method described in the present paper fulfils the first four criteria in respect of the single resistance character, namely resistance to aromatic arsenicals and amino-acridines.

Principles of the photosensitivity method. Acridine-induced photosensitivity in protozoa was first reported by Raab (1900) and elaborated by Tappeiner (1909) and Metzner (1924, 1927). Jansco (1931) observed that trypanosomes which were resistant to aromatic arsenical drugs, e.g. tryparsamide, were not as photosensitive as were normal trypanosomes. Jansco's observation confirmed the cross-resistance between acriflavine and aromatic arsenicals. Hawking (1988) measured the time in which normal and arsenic-resistant trypanosomes after incubation in acriflavine took to die when exposed to a bright patch of light in the centre of a dark-ground field. From the measurements it was apparent that variation existed between individuals. This was a simple method of estimating the mean value of a character in an infection and also the standard deviation of that character. However, it was necessary to use drug concentrations relevant to the degree of resistance in the strain being tested. Thus without further information relating the effect of light and the concentration of drug, comparison between strains was impossible. Further, owing to the difficulty in obtaining uniform illumination by dark ground a high value for the coefficient of variation was found.

In the method described here trypanosomes were incubated in various concentrations of acriflavine and then exposed to light. The time and intensity of exposure to light were made constant. After exposure the mortality rate was noted and its percentage calculated. The percentage mortalities were converted into probits before plotting a dose-response curve. For a single population the mean of individual sensitivities was given by the ED 50 and the reciprocal of the gradient was taken to be the standard deviation of that population. When two or more populations were present the dose-response curve became a characteristic ' $S$ ' shape. This will be described later, together with simple methods for analysis. 


\section{METHODS}

During the development of the method to be described, several factors which affect the photosensitivity reaction were examined. From the results of this preliminary investigation on photosensitivity per se it was possible to derive the practical method. The preliminary studies may be recorded in outline both for the information gained about photosensitivity and also because it has been found useful to calculate deviations caused by the inevitable slight alteration of the conditions which occur when a biological technique is used routinely.

Basic equipment. An early Leitz monocular microscope was mounted on a base board together with a $30 \mathrm{~W}(6 \mathrm{~V}, 5 \mathrm{~A})$. projection lamp, collector doublet lens, iris diaphragm, solenoid-operated camera shutter and the holder for a viewing filter. The normal microscope mirror was used and so also was the original aplanatic condenser. To make certain that no light from spurious reflexions or even light from the maximum aperture of the condenser reached the stage, a diaphragm was placed on top of the upper condenser lens. Slides were prepared from a suspension of trypanosomes after suitable incubation, by containing the organisms under a square cover slip ridged with petroleum jelly on three sides. It was of the greatest importance that no movement of the suspension should occur during the whole time a slide was being used. The optics above the stage were a Watson parachromatic objective $(\times 40)$ and a Zeiss $(\times 12)$ holoscopic ocular. The microscope tube was lined with black felt to decrease reflexions which otherwise impaired the image and caused the light meter readings to be unreliable.

Power for the projection lamp was obtained from a constant voltage transformer. As shown in the next section, the action spectrum for acriflavine-induced photosensitivity is in the blue-violet region. Tungsten filament lamps produce little energy in the blue-violet region and the amount is greatly dependent on the supply voltage. Other light sources such as high pressure mercury arc and 1000-W projection lamps were used but were less suitable than the low wattage tungsten lamp. The principal difficulty with the high-powered lamps was that the intense heat produced precluded the use of an efficient collector lens. In practice the most intense illumination was obtained by a short focal length $(f=4.7 \mathrm{~cm}$.), high aperture (4, $\mathrm{cm}$. diameter) collector lens and a $30 \mathrm{~W}$ lamp.

The solenoid-operated camera shutter was controlled by a thyratron timer. The device could be pre-set to give accurate exposures between 1 and $14 \mathrm{sec}$.

The viewing filter protected the trypanosomes and the operator from damage by the intense light. The filter consisted of a dense negative (optical density $=\mathbf{2 \cdot 3 5}$ ) and a deep red glass filter. The filter holder was arranged so that the filter could be slipped quickly in and out of the light path.

A light meter was used to standardize the intensity of illumination at the stage. When the optics were in Köhler illumination and normal focus was achieved, the eyepiece was removed and $a \times 6$ eyepiece with an attached photocell was inserted. The photocell (Mullard $90 \mathrm{AV}$ ) was chosen because its spectral response, through glass optics, has a peak sensitivity in the blue-violet region. A sensitive spotgalvanometer was used in the photocell circuit. The response of the photocell was tested and found to be linear to light intensity over the range of light intensities used. 
Strains of trypanosomes and rodent hosts. A monomorphic virulent strain of Trypanosoma brucei (NIMR 2) group was used for the development of the method. Strain NIMR 2 was fully adapted to rats and to mice by rapid syringe passage in alternate hosts. Trypanosoma rhodesiense EaTro 118 was obtained from the blood of infected rats, it was isolated from man in 1959 at the East African Trypanosomiasis Research Organization.Trypanosoma equiperdum (NIMR1) was used in one experiment, this strain came from Dr J. D. Fulton and was known originally as Wellcome oE (Fulton \& Spooner, 1959). NIMr numbered strains refer to the strains held by the trypanosome bank at the National Institute for Medical Research.

Male mice of the Parkes's strain were chosen because of the freedom from possible disturbance of the course of infection by the oestrous cycle of the female and also because this strain is extremely docile. Rats were of the hooded (black and white) strain and were all males for the same reasons.

\section{Factors which affect photosensitivity}

Simple tests of the effect of the temperature of incubation showed that no observable change in photosensitivity occurred between $4^{\circ}$ and $37^{\circ}$.

The temperature during exposure to light greatly altered the trypanosome mortality. The effect was examined by using a water-warmed slide and thermistor measuring device. Water was pumped in a closed system which consisted of two copper coils immersed in beakers of water and a space between two large coverslips. The test trypanosome suspension was held between two coverslips and was placed on the upper coverslip of the warmed slide with liquid paraffin making a heatconducting joint between the two glass surfaces. The temperature on top of theslide became that of the water within a few seconds. With this equipment it was possible to obtain accurately controlled and stable temperatures which could be altered to any new value within a minute or two, simply by adding hot water or ice to the beakers containing the heat-exchange coils. Observations of photosensitivity were made by the 'one concentration method' (see below, Analysis of results, for details) and the results adjusted according to the values found for each overlapping range in temperature. Experiments were made on different days. The results are expressed in Fig. 1. These show that relatively small changes, when compared with other factors, occurred up to $26^{\circ}$, but above this the photosensitivity increased more steeply. The plateau $35^{\circ}$ to $41^{\circ}$ and the sharp rise at higher temperatures are features about which nothing is known. The findings suggest that variations in 'laboratory temperature' $\left(20^{\circ}-22^{\circ}\right)$ would be insignificant for practical purposes.

Action spectrum. Experiments of Tappeiner (1909), Metzner (1924) and Jansco (1931) showed qualitatively that the action spectrum was in the region of peak absorption of acriflavine $(\lambda=454 \mathrm{~m} \mu)$. Ultraviolet radiation was used to find whether photosensitivity also occurred in the absorption band in this region. Light from a mercury vapour lamp passed through a Chance OX $\boldsymbol{\gamma}$ filter and was focussed by quartz optics on to a quartz slide. No trypanosomes were killed after exposure for $2 \mathrm{~min}$. in a concentration of acriflavine $1 \mu \mathrm{g} . / \mathrm{ml}$. It is concluded that ultraviolet radiation does not cause photosensitivity.

Drug concentration. The concentration of acriflavine was the variable chosen to be a measure of photosensitivity. The choice was made because the concentration can be known accurately and the procedure is easier, since logarithmic dilution 
series can be made up once, whereas a logarithmic series of light exposures cannot accurately be made.

Acriflavine was insufficiently soluble in Ringer's solution, and all experiments were done with acriflavine dissolved in ion-exchange water. A dilution by volume of one part of stock drug solution was made with nine parts of trypanosome suspension. Thus all results refer to a slightly hypotonic suspension.

Time of exposure to light and drug concentration. The interaction of these two variables was examined by using the ED 50 values for time for exposure as the endpoint. Trypanosome suspensions at several concentrations were used and the

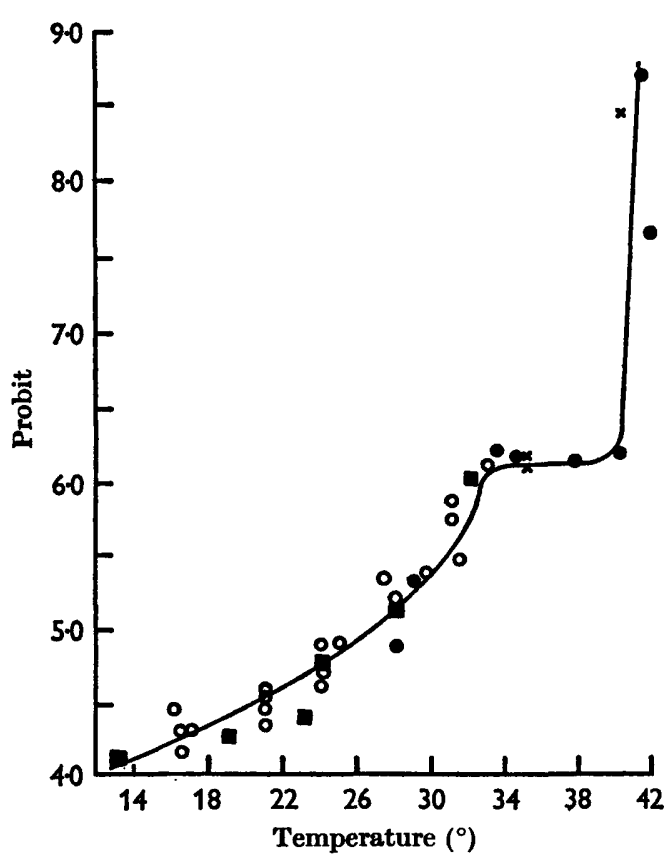

Fig. 1

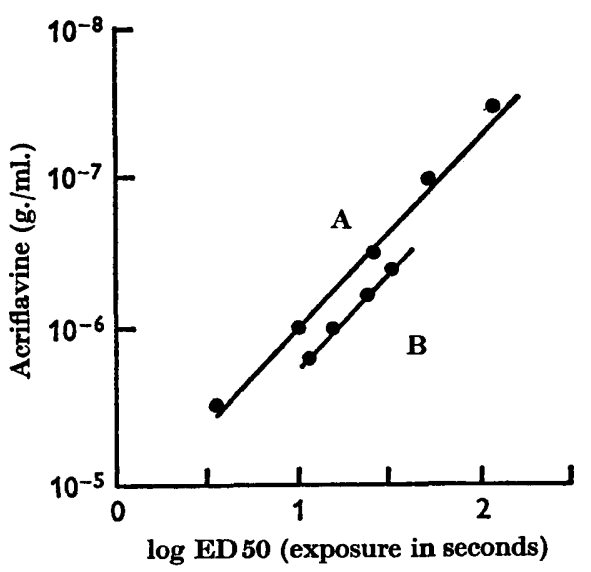

Fig. 2

Fig. 1. The effect of temperature on photosensitivity of organisms. Different symbols indicate experiments on different days. Three concentrations of acriflavine were $10^{-5.8}$, $10^{-6.0}$ and $10^{-6.3}$.

Fig. 2. The effect of concentration of acriflavine on the ED50 measured in seconds (see text).

mortality observed after four or five different exposure times at each concentration. The logarithm of the ED 50 (in seconds) for concentrations is plotted against the logarithm of the concentration in Fig. 2. Line A was drawn through points obtained over a wide range of drug concentrations and times of exposure. Line $B$ confirms the gradient of $\mathbf{A}$, both are 1.3 over a narrower range. $A$ systematic difference exists because of a change in the intensity of illumination between experiments.

The existence of a uniform relation between drug concentration and time of exposure was found for Trypanosoma equiperdum (NIMR 1). Similar, but less detailed experiments suggested that the gradient is characteristic of the strain. The gradients for T. brucei (NIMR 2) and T. rhodesiense (EATRO 118) were 1.0 and 0.91 , respectively. 
Time of incubation. Hawking (1934, 1938) found that uptake of acriflavine was very rapid, being virtually complete after a few minutes. This was not found in the present work, when spectrophotometric determinations of acriflavine remaining in solution were made. The results are shown in Fig. 3 and indicate that about $1 \mathrm{hr}$ was required to reach equilibrium.

Measurements of how the photosensitivity changed with time of incubation were made by using the one-concentration method. The results are shown in Fig. $4 a, b$. In both trypanosome strains the increasing photosensitivity had a value about equal to the steady value after $20 \mathrm{~min}$. However, between 20 and $40 \mathrm{~min}$. a peak sensitivity showed, only to fall to a steady value at $60 \mathrm{~min}$. Exposures made after prolonged incubation (up to $\mathbf{9} \mathrm{hr}$ ) showed no real change in the response.

These results suggest that for a practical method $1 \mathrm{hr}$ is the minimum period for incubation; after this it is not critical when the exposures are made.

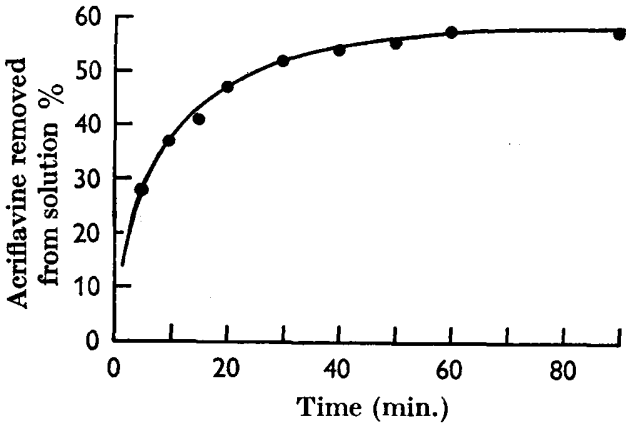

Fig. 3

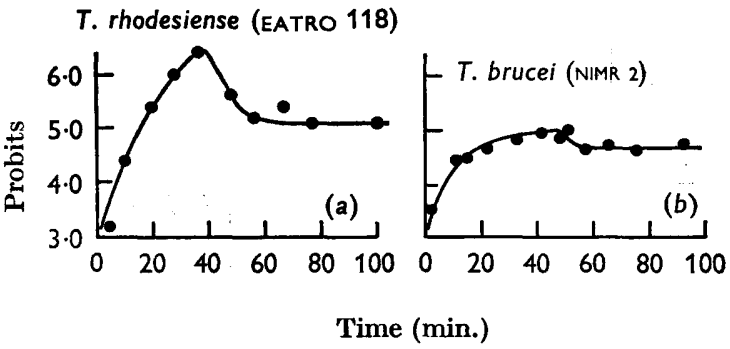

Fig. 4

Fig. 3. Spectrophotometric analysis of the rate of uptake of acriflavine by trypanosomes. Initial concentration $10^{-6 \cdot 0}$, trypanosomes $2000 / \mu \mathrm{l}$.

Fig. 4. The effect of time of incubation on photosensitivity. Concentration for Trypanosoma rhodesiense EATro 118 was $10^{-5 \cdot 8}$ and for $T$. brucei it was $10^{-6 \cdot 3}$.

The number of trypanosomes. Hawking (1938) observed that for a given concentration of drug when there were few trypanosomes these were more photosensitive than when there were many parasites. The present experiments confirmed this; ED 50 (in weight/volume units) was inversely proportional to the number of trypanosomes. It is therefore possible to correct mean values of photosensitivity for small departures from the standard number of trypanosomes.

The effect of serum and red cells. Serum and red cells are likely contaminants of trypanosome suspensions; their effects were examined by using the one-concentration method. The results from experiments with different concentrations of inactivated horse serum are given in Fig. 5. The results show that serum decreased the photosensitivity in a way roughly inversely proportional to the logarithm of concentration. For practical purposes it was necessary to use a medium which contained more serum than likely to be present as a contaminant. In this way the concentration of serum was standardized without detriment to the parasites by repeated centrifugation.

Red cells did not influence photosensitivity up to $5000 / \mu \mathrm{l}$. This number is more than is desirable for other reasons. 
The effect of $p H$ value. This factor was investigated by using isotonic phosphate buffers between pH 5.8 and 7.7 (Hendry, 1948) and analysed by the one-concentration method at two times of exposure. The results, shown in Fig. 6, emphasize the need for the accurate control of $\mathrm{pH}$ value. It is convenient that $\mathrm{pH} \mathbf{7 \cdot 2}$ was both at the peak of sensitivity and also was a suitable value for good longevity of parasites in suspension (Lumsden et al. 1963).

The effect of oxygen. Oxygen is stated to have a profound effect on photosensitivity (Blum, 1941; Reid, 1960). Trypanosomes were incubated; $(a)$ under liquid paraffin, (b) with the surface open to the atmosphere, and (c) with oxygen bubbled slowly through suspension. No differences were found between the treatments. In another

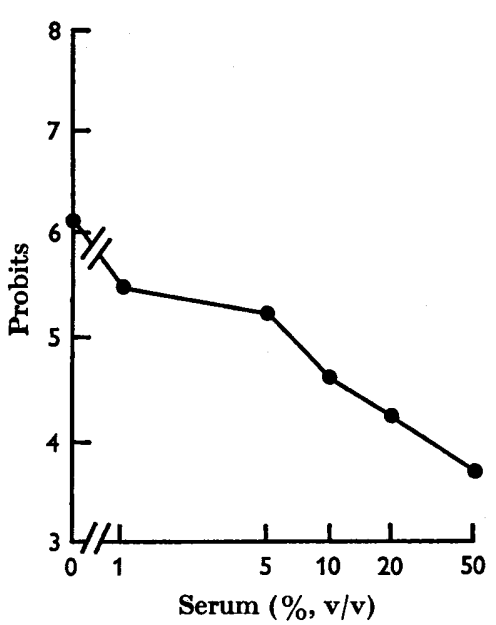

Fig. 5

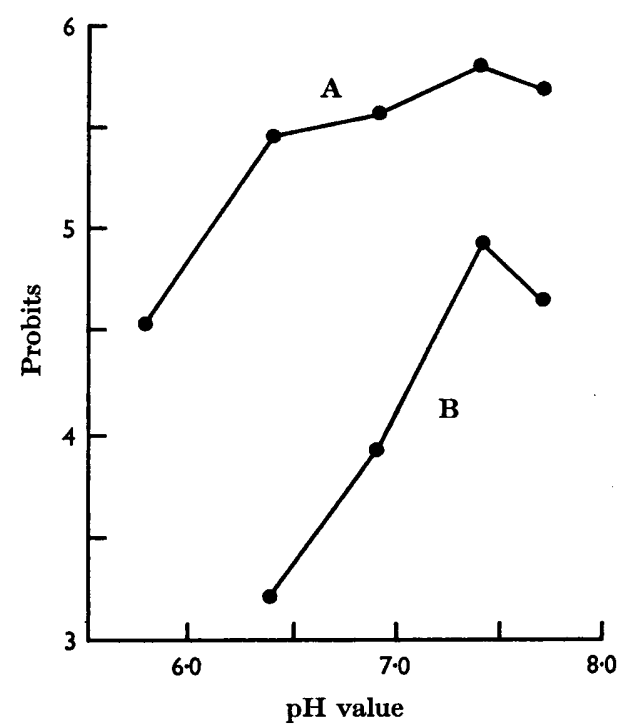

Fig. 6

Fig. 5. The effect of serum on photosensitivity.

Fig. 6. The effect of pH value on photosensitivity. A, exposure was $10 \cdot 6 \mathrm{sec}$. and $B$, exposure was $5 \cdot 5$ sec.

experiment suspensions of trypanosomes, which consume oxygen vigorously, were kept in sealed slides for up to $9 \mathrm{hr}$. The photosensitivity, which was expected to decrease through lack of oxygen, remained constant or increased very slightly. It was concluded that the oxygen tension during incubation and exposure exerted little or no effect on quantitative photosensitivity.

\section{The method for routine photosensitivity measurements}

Details of the apparatus have already been described. From consideration of the changes caused by various factors (above) the final method may now be described.

The standard buffered medium consisted of: $50 \mathrm{ml}$. inactivated $\left(56^{\circ}, 30 \mathrm{~min}\right.$.) horse serum (Oxoid); $300 \mathrm{ml}$. Hendry's phosphate buffer ( $\mathrm{pH} \mathrm{7 \cdot 2);37.5} \mathrm{ml.} \mathrm{isotonic}$ glucose (53.4 g./l.); $10 \mathrm{ml}$. phenol red solution (1/1000 in saline); made up to 11 . with Ringer's solution. This mixture was Seitz-filtered and put into $25 \mathrm{ml}$. containers; its keeping properties at $4^{\circ}$ appear to be good. 
Solutions of pure neutral acriflavine (3,6-diamino-10-methyl acridinium chloride; from Messrs Boots Ltd., Nottingham) were made in ion-exchange water because of the insolubility of the pure compound in balanced salts solutions. The initial concentration was $1 / 1000(\mathrm{w} / \mathrm{v})$ and serial tenfold dilutions were made. From each dilution were prepared nine others separated at a logarithmic interval of $0 \cdot 1$ until a continuous sequence $10^{-3 \cdot 0}, 10^{-3 \cdot 1}, 10^{-3 \cdot 2}, \ldots 10^{-4 \cdot 0}, 10^{4 \cdot 1}, \ldots 10^{-5 \cdot 0}, 10^{-5 \cdot 1}, \ldots$ to $10^{-5 \cdot 9}$ and $10^{-6 \cdot 0}$ was obtained. These solutions served as stock for all further experiments. The rack of tubes was covered with a light-tight hood and stored at $4^{\circ}$. No change in the activity of the acriflavine has been observed during 3 years.

To avoid cumbersome wording, the dilutions and concentrations of acriflavine will be expressed as the positive value of the negative logarithm. Thus $1 \mu \mathrm{g} . / \mathrm{ml}$. becomes $6 \cdot 0$, and $0.5 \mu \mathrm{g} . / \mathrm{ml}$. becomes $6 \cdot 3$. The advantages of this system of notation, which is similar to the pH scale, were discussed by Walker (1964).

Trypanosome suspensions were prepared by centrifugation of heavily infected heart blood taken from young rats (60-100 g.). Heparin at the rate of 1 in 5000 $(\mathrm{w} / \mathrm{v})$ was used. The layer of trypanosomes was dispersed slightly and transferred to a quantity of the medium. The number of trypanosomes was adjusted from successive haemocytometer counts until there were about $10,000 / \mu \mathrm{l}$.

Suitable acriflavine solutions were delivered in $0.1 \mathrm{ml}$. lots into each of a row of small glass tubes and $0.9 \mathrm{ml}$. of the trypanosome suspension was added to each tube. The tubes then were quickly shaken and the rack covered with a hood to exclude light. The preceding manipulations were all made in very dim light.

Incubation was for $1 \mathrm{hr}$ at $20^{\circ}$ to $22^{\circ}$ (room temperature). Each tube was well shaken before a capillary tube was used to remove a small quantity of suspension from it. The drop of suspension was placed on a coverslip ridged on three sides with petroleum jelly. A slide (thickness $0 \cdot 88-0.95 \mathrm{~mm}$.) was lowered on to the coverslip. The preparation was examined by using the observation filter to check that it was not still flowing, and a field containing 40-60 trypanosomes was then located.

The microscope had previously been adjusted by using a standard slide to give a deflexion of 9 units on the galvanometer scale. Calculation, based on the manufacturer's data for the photocell, shows that about 0.064 lumens were passing into the eyepiece field of $\mathbf{3 7 2} \mu$ diameter. Neglecting the absorption at the glass surfaces the intensity experienced by the trypanosome was roughly $5 \cdot 8 \times 10^{-7}$ lumens $/ \mu^{2}$ or, if the flagellum is considered as the only photosensitive organelle (Walker, 1961), then in $10 \mathrm{sec}$. the flagellum receives a total of $2 \cdot 2 \times 10^{-2}$ ergs or $5 \times 10^{9}$ photons in each $\mu$ of its length. This calculation uses the value $0.25 \mu$ as the diameter of the flagellum (Dr K. Vickerman and Dr J. Williamson, personal communications).

Exposure without the observation filter in all routine experiments was for 10 sec. With the filter replaced the dead organisms were counted. A trypanosome was considered as dead when, after 1 min., no wriggling movements were seen. Experience showed that a few trypanosomes were motionless immediately after exposure but moved slightly within 10-30 sec. Prolonged observation of individual trypanosomes suggested that when no movement occurred after more than 30 sec. the organism would not recover motility. Two exposures were usually made for each preparation. The proportions of organisms killed were usually close in both exposures; but when they were not, a third or fourth exposure was made, and the proportions in close agreement were used for calculation. Data from each exposure of the number 
killed and of the total number were added together and expressed as a percentage. The percentage was converted into a probit number by using tables, and the probit numbers plotted on the ordinate against drug concentration in logarithmic units. A complete dose-response curve was thus built up by using the number of drug concentrations necessary to cover the range of sensitivity of a given strain (Figs. 7, 8). While it is a simple matter to see an occasional living trypanosome among many dead ones and to count them accurately, the reverse situation is not so easy. This practical difficulty caused the data below probit $3 \cdot 7$ to be less accurate than other points.

Counts were made of trypanosome suspensions by adding $0.1 \mathrm{ml}$. of a $4 \mathrm{mg} . / \mathrm{ml}$. HCHO in isotonic saline with $0.5 \%$ methylene blue to $0.9 \mathrm{ml}$. of suspension. Ten large squares of an improved Neubauer haemocytometer were counted. These squares contained a total of about $\mathbf{4 0 0}$ trypanosomes. The expected standard error of counts was $5 \%$.

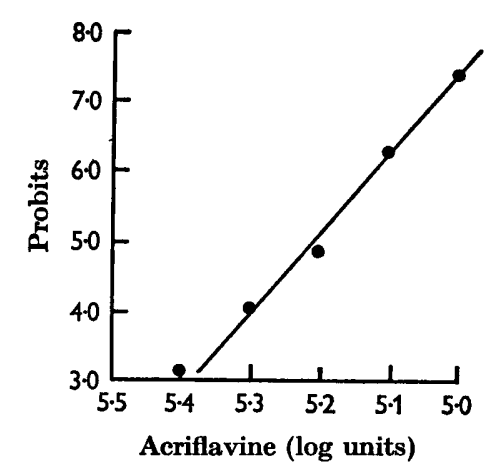

Fig. 7

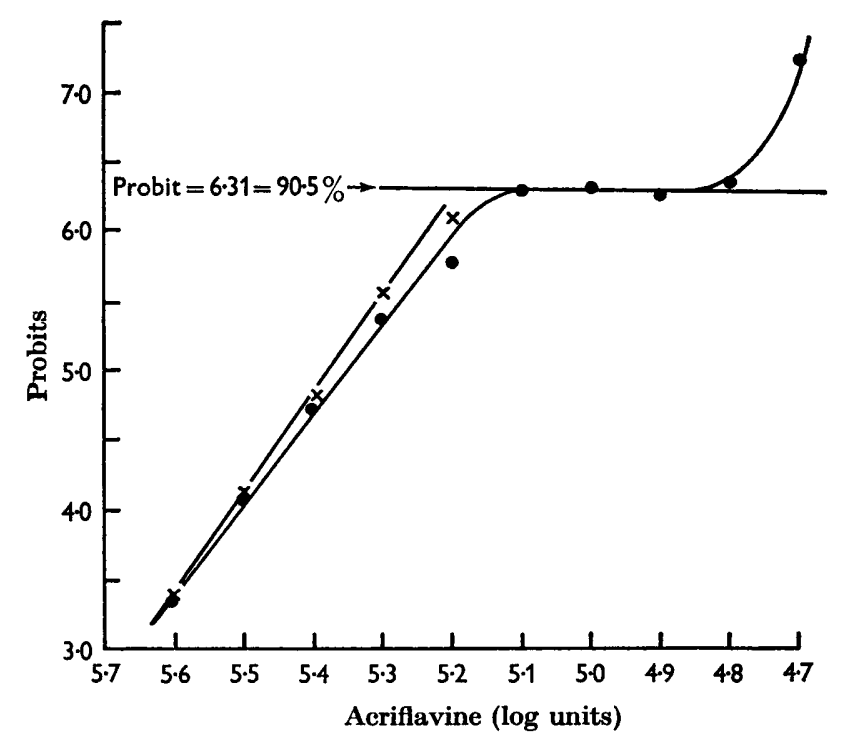

Fig. 8

Fig. 7. Results from the photosensitivity analysis of a resistant homogenous (log-normal) strain of Trypanosome brucei (NIMR 2.) For calculation of log units of acriflavine see text p. 52.

Fig. 8. Results from the photosensitivity analysis of a resistant infection of Trypanosoma brucei (NIMR2) which contained two populations (see Table 1). O, Experimental points. $\times$, recalculated points for major population (see text).

\section{Analysis of results}

Analysis of data from routine tests was performed graphically by fitting the best straight line or curve to a set of points (Fig. 7). If the strain consisted of one population, the ED 50 value and standard deviation were read directly in the usual way. Correction for departure from the standard number of trypanosomes was made by adding or subtracting the difference between the logarithms of the number of trypanosomes and the standard $(10,000 / \mu \mathrm{l}$.). Thus when too few trypanosomes were present the ED 50 was larger, and it was necessary to subtract the correction for 
number. This process is valid since both the difference in number and the drug concentration are expressed in logarithms to the base 10 .

In the development of drug resistance several populations were found frequently in the same infection. A graph of probit $v s$ log concentration appeared as a rounded step or steps (Fig. 8). The flat portion or point in inflexion between each steeply rising section of a curve indicated the probit which corresponded to the percentage of the total infection where one population ended and another began (Harding, 1949). Analyses of these inflexed curves were made graphically. When two populations were found the flat region of the curve was extrapolated to the ordinate and the probit obtained. The percentage mortality which corresponded to this probit indicated the proportion of the infection occupied by the more sensitive population. The percentage occupied by the less sensitive population was obtained by subtraction from 100. Because of the distortions introduced when two populations existed together it was necessary to recalculate the percentage mortality at each concentration and express the figure as a percentage of the relevant population. These new percentages were replotted as probits and the two straight lines analysed separately. Frequently one resistant population represented less than $5 \%$ of the total infection and it was impossible to give accurate evaluation to either ED 50 or standard deviation, although from the shape of the curve the existence of the population was certain. In such cases it was only possible to state the range of concentrations in which such a small population was found.

When tests were made by varying some factor and measuring the effect on photosensitivity the normal Trypanosoma brucei (NIMR 2) strain was used. This strain was known to give consistent results and to have a log-normal distribution of sensitivity which, of course, appeared normal because the drug concentrations were made up on a logarithmic scale. It was therefore possible to use the relation that for a given strain: $P_{2}-P_{1}=\left(C_{1}-C_{2}\right) / s$, when $P_{1}, P_{2}$ are observed probit values, $C_{1}, C_{2}$ concentrations of acriflavine (in the logarithmic scale) and $s$ is the standard deviation of sensitivity in the population. It follows that when one concentration is used the probit can be taken as indicating photosensitivity. This greatly decreased the number of observations needed and simplified the analysis.

When one factor caused a very wide variation in photosensitivity (e.g. the effect of temperature; Fig. 1) three concentrations were used. The final graph was constructed by taking one concentration as a standard and then calculating the difference between the probit values when temperatures overlapped. The difference was used to adjust the observations from the second and third concentrations in terms of the first concentration. The graph (Fig. 1) still bearing the probit scale looks absurd, for a probit value of $\mathbf{9 \cdot 0}$ is impossible to measure directly.

\section{RESULTS}

\section{Single populations}

Typical results for a resistant Trypanosoma brucei (NIMR 2) are shown in Fig. 7. This straight line was fitted by eye after having made allowance for the loss of weight as points depart from the ED 50 (probit 5). The ED 50 from the graph was $5 \cdot 21$, standard deviation 0.09 . The suspension of trypanosomes used in this experiment contained $39 \cdot 3 / \mu$ l. and this gives a correction of 0.01 to be subtracted from the 
ED 50. The corrected mean photosensitivity for the experiment on this (resistant) strain was therefore 5.20; S.D. 0.09. These observations suggest this strain has a normal distribution of sensitivity and it is unlikely to have more than $4.5 \%$ of another population. Estimates of the maximum value for an unseen population are obtained from the Poisson distribution of the mean number of resistant trypanosomes per two fields when $100 \%$ mortality would be expected 19 times in 20 . The percentage is found from: maximum percentage not seen $=5 / x$ when $x$ is the total number of trypanosomes at the first concentration at which all are killed.

\section{Two populations in a single infection}

Trypanosome strain c 5 was obtained during an experiment on the development of resistance to aromatic arsenical compounds in Trypanosoma brucei (NIMR 2). A graph of the results is shown in Fig. 8. The flat region of the curve is at probit 6.31 and it represents $\mathbf{9 0 . 5 \%}$ of the infection. A second analysis of the major population is also shown in Fig. 8. The features of each population are given in Table 1.

Table 1. Analysis of the populations of a strain of Trypanosoma brucei (NIMR 2) with a bimodal distribution of sensitivity to acriflavine after exposure to a constant quantity of light

$\begin{array}{lcccc} & \text { \% of total } & \text { ED 50 } & \begin{array}{c}\text { Corrected } \\ \text { ED 50 }\end{array} & \begin{array}{c}\text { Standard } \\ \text { deviation } \\ \text { or range }\end{array} \\ \text { Population A } & 90.5 & 5.375 & 5 \cdot 35 & 0 \cdot 14 \\ \text { Population B } & 9.5 & 4.75 & 4.73 & 4.7-4.8\end{array}$

See text for the units of the ED 50 and method of correction.

Since fewer trypanosomes are represented in population $\mathbf{B}$, the accuracy of its ED 50 value was less than for population $A$. The standard deviation for population $B$ cannot be calculated with certainty from such data. It is thought better to give a range which included 'most' of the population. It would be ideal if 'most' covered $\pm 2 s(95 \%)$ of the population; but this is not possible and all attempts at pseudoaccuracy have been avoided.

Standard errors of the ED 50 can be found in the way adapted for probit analysis and comparison can be made between the populations. In practice a rule-of-thumb test, which is only applicable to strains with characteristics similar to those given above, is possible since the standard error of each ED 50 is given approximately by $s / \sqrt{ }(1 \cdot 5 n)$, where $s$ is the standard deviation and $n$ the mean number of trypanosomes per concentration present in the population being analysed. For populations as small as B (Table 1) no excessive claims for fine analysis arise when a figure of half the range is used for the standard error.

\section{Accuracy of analyses of Trypanosoma brucei (NIMR 2) populations}

Replicate analyses were made early in the development of the technique. The strain of Trypanosoma brucei (NIMR 2), was chosen because of its known sensitivity to arsenical drugs and because analyses had suggested it was a single population of normal sensitivity. The ED 50 values are compared in Table 2. Three analyses A, $\mathrm{B}$ and $\mathrm{C}$ were made one after the other. At each concentration two exposures were 
made (numbered 1 and 2). The results from exposures 1 and 2 were combined to give data in $\mathrm{A} 1+2, \mathrm{~B} 1+2, \mathrm{C} 1+2$. The results from all exposures at each concentration were combined and analysed. This is shown in the row 'combined results'. In Table 2 the results given in the column headed ' graphical analyses' were obtained without correction for number of trypanosomes from the graphs. Arithmetical analyses were made by Miss M. V. Mussett, who used the usual re-iterative method for probits.

Table 2. Table of ED 50 and $s$ obtained by graphical and arithmetical methods of analysis of normally sensitive Trypanosoma brucei (NIMR 2)

\begin{tabular}{|c|c|c|c|c|c|}
\hline \multirow[b]{3}{*}{ Group } & \multicolumn{5}{|c|}{ Method of analysis } \\
\hline & \multicolumn{2}{|c|}{ Graphical } & \multicolumn{3}{|c|}{ Arithmetical } \\
\hline & ED 50 & $s$ & ED 50 & $\boldsymbol{s}$ & $\boldsymbol{P}$ \\
\hline Al & $6 \cdot 30$ & $0 \cdot 19$ & $6 \cdot 308$ & $\mathbf{0} \cdot \mathbf{1 6 3}$ & 0.4 \\
\hline A2 & $6 \cdot 28$ & 0.20 & 6•294 & $0 \cdot 193$ & $0 \cdot 8$ \\
\hline B 1 & $6 \cdot 28$ & $0 \cdot 16$ & $6 \cdot 277$ & $0 \cdot 158$ & $\mathbf{0} \cdot \mathbf{3}$ \\
\hline B2 & $6 \cdot 28$ & $0 \cdot 16$ & $6 \cdot 284$ & $0 \cdot 174$ & $0 \cdot 8$ \\
\hline $\mathrm{C} 1$ & $6 \cdot 27$ & $0 \cdot 16$ & $6 \cdot 266$ & $\mathbf{0} \cdot \mathbf{1 3 9}$ & 0.4 \\
\hline $\mathrm{C2}$ & $6 \cdot 24$ & $0 \cdot 18$ & $6 \cdot 252$ & $0 \cdot 143$ & 0.2 \\
\hline $\mathbf{A 1}+\mathbf{2}$ & $6 \cdot 28$ & $0 \cdot 209$ & $6 \cdot 305$ & $0 \cdot 184$ & $0 \cdot 6$ \\
\hline $\mathrm{B} 1+2$ & $6 \cdot 28$ & $0 \cdot 169$ & $6 \cdot 281$ & $0 \cdot 166$ & $\mathbf{0 . 3}$ \\
\hline $\mathrm{C} 1+2$ & $6 \cdot 25$ & $0 \cdot 188$ & $6 \cdot 258$ & $0 \cdot 143$ & $0 \cdot 1$ \\
\hline Combined results & $6 \cdot 287$ & $0 \cdot 181$ & $6 \cdot 285$ & $0 \cdot 172$ & $0 \cdot 8$ \\
\hline
\end{tabular}

$s=$ standard deviation of population.

$P=$ probability of being derived from a homogenous population (from $\chi^{2}$ for linearity).

Table 3. Results of photosensitivity analysis made on Trypanosoma brucei, (NIMR 2)

\begin{tabular}{|ccc} 
Date & ED 50 & $\begin{array}{c}\text { Standard } \\
\text { deviation }\end{array}$ \\
15 June 61 & $6 \cdot 14$ & $0 \cdot 14$ \\
28 Aug. 61 & $6 \cdot 07$ & $0 \cdot 11$ \\
2 Sept. 61 & $6 \cdot 17$ & $0 \cdot 12$ \\
17 Oct. 61 & $6 \cdot 05$ & $0 \cdot 16$ \\
26 Oct. 61 & $6 \cdot 10$ & $0 \cdot 10$ \\
18 Nov. 61 & $6 \cdot 06$ & $0 \cdot 07$ \\
15 Nov. 62 & $6 \cdot 14$ & $0 \cdot 15$ \\
29 Nov. 62 & $6 \cdot 08$ & $0 \cdot 17$ \\
7 Dec. 62 & $6 \cdot 11$ & $0 \cdot 17$ \\
13 Dec. 62 & $6 \cdot 12$ & $0 \cdot 09$ \\
27 Nov. 63 & $6 \cdot 08$ & $0 \cdot 04$ \\
28 Nov. 63 & $6 \cdot 12$ & $0 \cdot 10$ \\
29 Nov. 63 & $6 \cdot 07$ & $0 \cdot 05$ \\
5 Dec. 63 & $6 \cdot 08$ & $0 \cdot 15$
\end{tabular}

The results analysed by either method show considerable uniformity even between the primary observations, A 1, B 1, etc., which represent a group size of only 40 trypanosomes. Since nearly $4 \mathrm{hr}$ elapsed between the first and last measurements the temporal stability of the character is clearly indicated. The only consistent difference between the graphical and arithmetical methods of analysis appears in the estima- 
tion of standard deviation. This difference is due to considerable loss of weight when points depart from probit 5 . The discrepancy is small and for the present purposes unimportant.

\section{Stability of drug sensitivity of Trypanosoma brucei (NIMR 2)}

Photosensitivity observations made over some years are presented in Table 3. Although it is difficult to distinguish between instability of a character from instability of the system of measuring it, the results suggest stability in both the strain and the method. The figures given in Table 3 may be compared with the lowest degree of stable resistance which has a value of $5 \cdot 2$ units.

\section{DISCUSSION}

The results obtained by using acriflavine-induced photosensitivity show clearly that the method is capable of producing reliable estimates which fufil most of the criteria set out in the Introduction. It remains to discuss a few details which have not been tested by direct experiment and to comment on the reasons for adopting an elementary statistical treatment.

Oxygen tension has been shown to be without effect on photosensitivity despite biological removal or physical excess. For practical purposes, providing that the same manipulative procedures are followed, it would be impossible to exceed the extremes tested. However, removal of oxygen by addition of reducing compounds seems likely to interfere with the photosensitive reaction by direct action on the trypanosomes as well as by removing oxygen. Objections can be raised.to an alternative method of removing oxygen from a suspension by a stream of nitrogen, because of the very low concentration of oxygen which would be expected to take part in the reaction. It remains therefore uncertain whether oxygen is required in the photosensitive reaction. The indication, bearing in mind the high oxygen consumption of trypanosomes (Brand, 1952), is that the organisms are themselves capable of removing oxygen from an environment. If this is correct then the stable photosensitivity found in slides sealed for many hours suggests that oxygen plays no part in the reaction.

It was not possible to investigate changes in photosensitivity which might occur with age or heaviness of infection, because of the difficulty of preparing trypanosomes from light infections. In several experiments it was necessary to use blood from rats which were only showing ten trypanosomes $/ \times 40$ microscope field and from moribund rats with between 100 and 200 trypanosomes $/ \times 40$ field. No difference was detected between these analyses and trypanosomes from an intermediate degree of infection. It is presumed that the age and severity of an infection has no appreciable effect on photosensitivity.

The reasons for adopting a graphical standard of analysis are that time is saved and that, because of the large number of trypanosomes in each group, even the simplest analyses themselves have small errors and the complex refinements for probit analysis on small groups are unnecessary. It should be added that it is not possible at present to control the light intensity better than $2 \%$, with possibly wider fluctuations of the intensity of the active wavelength. This error may contribute to a variation of 0.03 units even in the same day.

For the purposes of example, if an average value for the standard deviation is 
taken to be $0 \cdot 1$ units and if an analysis has 5 points on probits $3,4,5,6$ and 7 , a significant difference $(P=0.05)$ is found between means with a separation ranging from 0.043 when $n=25$ to 0.020 when $n=100$. It is clear that while extrinsic errors such as those introduced by the intensity of illumination may be as large as 0.03 units, no useful purpose is served by increasing $n$ beyond 100. Although $n$ can be made smaller than 100 in theory, two exposures at one slide have beenfound necessary, since each exposure serves to check the other. Thus data based on two exposures have a combined group size within the range 60 to 110 trypanosomes.

The magnitude of variation in the sequence of measurements on Trypanosoma brucei (NIMR 2) (Table 3) is clearly significant when rigorous standards are applied. Not enough experiments have been done to enable an analysis of variance to be made. The range in this sequence is 0.12 units and in the opinion of the writer a difference between means of 0.12 units may be a conservative estimate of a significant difference. However, when populations which have widely different sensitivities are present in the same infection competitive uptake of drug must be expected. The effect of this is to make resistant populations appear a little more resistant than when they appear alone. It is concluded that although the photosensitivity method is adequate for the purpose intended, rigorous statistical tests of the results are inappropriate because of some non-random variation in mean photosensitivities.

\section{REFERENCES}

Blum, H. F. (1941). Photodynamic Action and Diseases Caused by Light. New York: Reinhold Publishing Corpn.

Brant, T. von (1952). Chemical Physiology of Endoparasitic Animals. New York: Academic Press.

Fulton, J. D. \& Spooner, D. F. (1959). Terminal respiration in certain mammalian trypanosomes. Expl. Parasit. 8, 137.

HARDING, J. P. (1949). The use of probability paper for the graphical analysis of polymodal frequency distributions. J. mar. biol. Ass. U.K. 28, 141.

Hawking, F. (1934). The absorption of acriflavine by trypanosomes. Ann. trop. med. Parasit. 28, 67.

Hawking, F. (1938). A quantitative study of the photosensitivity induced in trypanosomes by acriflavine. Ann. trop. med. Parasit. 32, 367.

Ha wring, F. \& Walker, P. J. (1966). Analysis of the development of arsenical resistance in trypanosomes in vitro. Expl. Parasit. 18, (in the press)

Hendry, E. B. (1948). Iso-osmotic phosphate buffer solutions. Edinb. med. J. 55, 142.

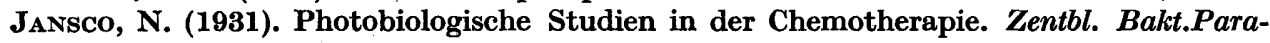
sitkde. Orig. 122, 388.

Lumsden, W. H. R., Cunningham, M. P., Webber, W. A. F., van Hoeve, K. \& Walker, P. J. (1963). A method for the measurement of the infectivity of Trypanosome suspensions. Expl. Parasit. 14, 269.

MetzNer, P. (1924). Zur Kenntnis der photodynamischen Erscheinung. III. Mitteilung: Ǔber die Bindung der wirksamen Farbstoffe in der Zelle. Biochem. Z. 148, 498.

Metziner, P. (1927). Photodynamische Erscheinung. Tabul. biol. 4, 496.

RAAB, O.v. (1900). Über die Wirkung fluoreszierender Stoffe auf Infusorien. Z. Biol. 39, 524.

REID, C. (1960). Quantum phenomena in biology. Science, N.Y. 131, 1078.

Tappeiner, H. v. (1909). Die photodynamische Erscheinung (Sensibilisierung durch fluoreszierende Stoffe.) Ergebn. Physiol. 8, 698.

WALKER, P. J. (1961). Organisation of function in trypanosome flagella. Nature, Lond. $189,1017$.

WALKER, P. J. (1964). Reproduction and heredity in trypanosomes. Int. Rev. Cytol. 17. 51. 